\title{
SPAWNING KINETICS OF INDIVIDUAL FEMALE MEAGRE (ARGYROSOMUS REGIUS) AFTER TREATMENT WITH GnRHa IMPLANTS
}

\author{
Mylonas, C.C.*, Mitrizakis, N.*o, Sigelaki, I.* and Papadaki, M.* \\ * Institute of Aquaculture, Hellenic Center for Marine Research, AQUALABS, P.O.Box 2214, Iraklion, Crete 71003, \\ Greece. Fax +302810337875 email: mylonas@her.hcmr.gr \\ ○ University of Crete, Biology Department, Heraklion, Crete 71409, Greece
}

\section{Introduction:}

The meagre (Argyrosomus regius) is a new candidate species for European aquaculture [1]. Though broodstocks are maintained by many commercial operations, this species does not spawn spontaneously in captivity, necessitating the development of spawning induction protocols. The present study reports on the use of agonists of gonadotropin releasing hormone (GnRHa) loaded in controlled-release implants for the induction of oocyte maturation and spawning of meagre. Both group spawnings and individual-female spawnings were induced, in order to obtain accurate information on the spawning kinetics and fecundity of this species.

Methods:

Three different spawning induction trials were conducted between May and June 2009. Males and females $(\mathrm{n}=10$, mean body weight $\pm \mathrm{SD}$ of $6.9 \pm 1.9 \mathrm{Kg})$ were implanted with EVAc GnRHa implants [2] at a dose of approximately $50 \mu \mathrm{g} \mathrm{Kg}^{-1}$ body weight and placed in 9000-1 tanks connected to overflow egg collectors. In 2010, six individual females were placed in separate 5000-1 tanks with two males each (mean body weight of $8.2 \pm 2.2 \mathrm{Kg}$ ) during May and June. From every spawning event, an egg sample was collected for the estimation of fecundity and fertilization success. Results were analyzed by ANOVA, followed by Duncan's New Multiple Range test (DNMR), at a minimum significance of $\mathrm{P}<0.05$.

Results:

Spawning was initiated 2 or 3 d after GnRHa treatment. In the group spawnings, mean relative fecundity spawn $^{-1}$ ranged from 24,300 to 49,900 eggs $\mathrm{Kg}^{-1}$ female body weight and mean fertilization success ranged from 85 to $87 \%$. The number of spawns was between 7 and 17 and mean total annual relative fecundity was $365,000 \pm 107,000$ eggs $\mathrm{Kg}^{-1}$ year $^{-1}$. No statistically significant difference was found in relative fecundity or fertilization success between the three different trials in 2009. In the individualized trials in 2010, the number of spawns per female ranged between 5 and 19, relative fecundity spawn $^{-1}$ ranged between 28,540 and 59,380 eggs $\mathrm{Kg}^{-1}$ and fertilization success ranged between 85 to $95 \%$ (Table 1).

Relative fecundity was maximal on the second or third spawn and then decreased gradually (Fig. 1). On the contrary, fertilization percentage was high throughout the spawning season. The majority of the eggs $(70 \%)$ were produced during the first four

Figure 1. Daily relative fecundity $\left(\mathrm{x} 10^{3} \mathrm{eggs} \mathrm{Kg}^{-1}\right.$ female biomass) and fertilization success (\%) of successive spawnings of a single female meagre after GnRHa implantation on June 3, 2010. The arrow refers to the day of the implantation.

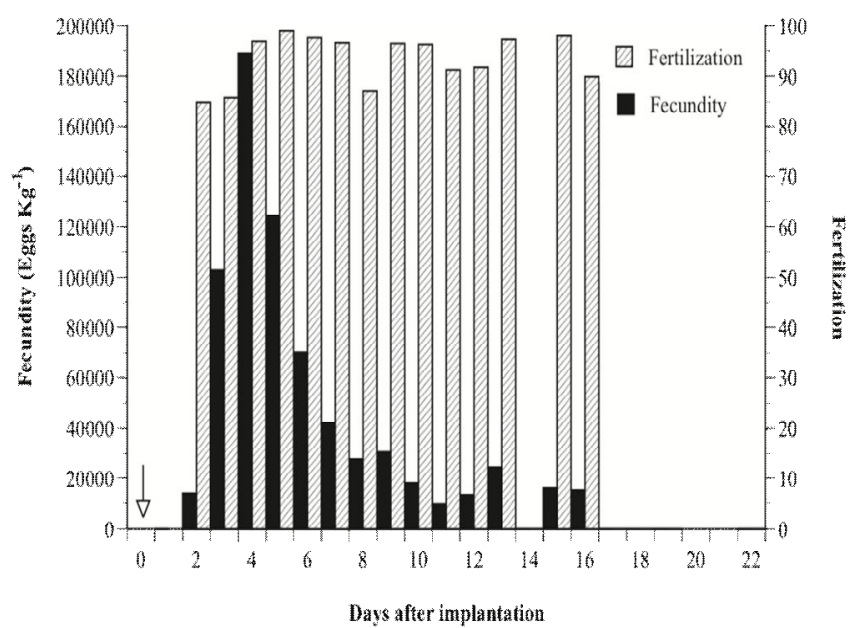

Table 1. Number of spawns, relative fecundity $\operatorname{spawn}^{-1}$, total annual relative fecundity and fertilization percentage of the two spawning induction trials conducted in 2010.

\begin{tabular}{|l|l|l|l|l|l|}
\hline $\begin{array}{l}\text { No } \\
\text { female }\end{array}$ & Date of implantation & Spawns & Eggs/ Kg/ Spawn & Eggs/ Kg/ Season & Fertilization (\%) \\
\hline 1 & $05 / 04 / 2010$ & 5 & 33,625 & 168,127 & 89 \\
\hline 2 & $05 / 04 / 2010$ & 10 & 28,540 & 285,401 & 85 \\
\hline 3 & $05 / 04 / 2010$ & 6 & 59,381 & 356,286 & 87 \\
\hline 4 & $06 / 03 / 2010$ & 14 & 49,957 & 699,394 & 93 \\
\hline 5 & $06 / 03 / 2010$ & 10 & 52,207 & 522,066 & 95 \\
\hline 6 & $06 / 03 / 2010$ & 19 & 30,415 & 577,886 & 95 \\
\hline
\end{tabular}


spawning events. No

statistically significant difference was observed in daily fecundity or fertilization success, either between individual females or between different GnRHa implantation dates.

\section{Discussion:}

The present study demonstrated that GnRHa implants are very effective in inducing spawning in the meagre, resulting in a large number of consecutive spawns, promoting the asynchronous nature of oocyte maturation of the species. No significant differences in egg production or quality were observed between group and individual spawnings, which is contrary to what is observed in other fish, which often do not reproduce well outside a large group [3]. Meagre can respond well to hormonal induction from May to June with the same effectiveness and egg quality, resulting in a mean annual fecundity of approximately 410,000 eggs $\mathrm{Kg}^{-1}$ and a mean fertilization percentage of $>75 \%$. The results obtained in the present study will be useful for the broodstock management of meagre in commercial operations, helping hatchery managers plan better and more efficiently their production.

\section{References:}

[1]QUÉMÉNER, L., SUQUET, M., MERO, D., GAIGNON, J.-L. 2002. Selection method of new candidates for finfish aquaculture: the case of the French Atlantic, the Channel and the North Sea coasts. Aquat. Living Resour., 15: 293-302

[2]MYLONAS, C. C., ZOHAR, Y. 2001. Use of GnRHa-delivery systems for the control of reproduction in fish. Rev. Fish Biol. Fish., 10: 463491.

[3]FORNIÉS, M. A., MAÑANOS, E., CARRILLO, M., ROCHA, A., LAUREAU, S., MYLONAS, C. C., ZOHAR, Y. ZANUY, S. 2001. Spawning induction of individualised European seabass females (Dicentrarchus labrax) using different GnRHadelivery systems. Aquaculture, 202: 221-234. 